\title{
On the eigenstructure of a class of max-plus linear systems*
}

\author{
G.A.D. Lopes, B. Kersbergen, T. van den Boom, B. De Schutter, and \\ R. Babuška
}

If you want to cite this report, please use the following reference instead:

G.A.D. Lopes, B. Kersbergen, T. van den Boom, B. De Schutter, and R. Babuška, "On the eigenstructure of a class of max-plus linear systems," Proceedings of the 2011 50th IEEE Conference on Decision and Control and European Control Conference (CDC-ECC), Orlando, Florida, pp. 1823-1828, Dec. 2011.

Delft Center for Systems and Control

Delft University of Technology

Mekelweg 2, 2628 CD Delft

The Netherlands

phone: +31-15-278.24.73 (secretary)

URL: https: / / www.dcsc.tudelft.nl

*This report can also be downloaded via https://pub. deschutter.info/abs/11_038.html 


\title{
On the eigenstructure of a class of max-plus linear systems
}

\author{
G.A.D. Lopes, B. Kersbergen, T. van den Boom, B. De Schutter, and R. Babuška
}

\begin{abstract}
Various applications in scheduling, such as train timetables and multi-legged locomotion, can be modeled using systems of max-plus linear equations. In this framework, the eigenvalue of the system matrix represents the total cycle time, whereas the eigenvector dictates the steady state behavior. For a class of concurrent two-state cyclic systems, with direct application to legged locomotion, we present closed-form expressions for the eigenvalue and eigenvector of the system matrix. Additionally, we probe into the transient properties of this class of max-plus linear systems by computing the coupling time.
\end{abstract}

\section{INTRODUCTION}

Max-plus linear discrete-event systems (MPL-DES) are a subclass of timed DES (classes of discrete event systems where there exists an underlying time structure) that can be framed in systems of linear equations in the max-plus algebra [1], [2], [3]. DES that enforce synchronization can be modeled in this framework. MPL systems inherit a large set of analysis and control synthesis tools thanks to many parallels between the max-plus-linear systems theory and the traditional linear systems theory. At the time of writing, the theory of max-plus algebras has been successfully applied to railroads [4], [5], queuing systems [6], resource allocation [7], and recently image processing [8] and legged locomotion [9], [10]. This paper continues the authors' application of max-plus systems to legged locomotion by investigating the structural properties of the system matrix.

Legged systems are traditionally modeled as limit cycles in cross products of circles in the phase space of the set of continuous time gaits (see Holmes et al. [11] for an extensive review on the elements of dynamic legged locomotion). In [10] we have introduced an abstraction to represent the combinatorial nature of the gait space for many-legged robots into ordered sets of leg index numbers. This abstraction allows for a systematic and straightforward implementation of motion controllers for many-legged robots. In this paper we present closed-form expressions for the eigenvalue and eigenvector of the system matrix for a class of max-plus linear systems. Such results can be utilized to compute the coupling time which in turn allows us to predict how many steps does a robot need to take to achieve steady-state after a gait switch or a large perturbation.

We start by revisiting in Section II the theory of maxplus algebras and in Section III we demonstrate how legged locomotion can be modeled by max-plus linear systems. In Section IV we present closed-form expressions for the

All authors are with the Delft Center for Systems and Control, Delft University of Technology, The Netherlands, e-mail: \{g.a.d.lopes, a.j.j.vandenboom, b.deschutter, r.babuska\}@tudelft.nl, b.kersbergen@student.tudelft.nl eigenvalue and eigenvector of the system matrix and in Section V we compute the coupling time.

\section{MAX-Plus AlGEBRA}

Max-plus algebras were introduced in the sixties by Giffler [12] and Cuninghame-Green [13]. In the late seventies the second author wrote the first book [1], and in the eighties Cohen et al. [14] presented a system-theoretic view. A few additional books have been published on the topic including [2], [3]. For a historical overview see [15]. The structure of the max-plus algebra is as follows: let $\varepsilon:=-\infty, e:=0$, and $\mathbb{R}_{\max }=\mathbb{R} \cup\{\varepsilon\}$. Define the operations $\oplus, \otimes: \mathbb{R}_{\max } \times$ $\mathbb{R}_{\max } \rightarrow \mathbb{R}_{\max }$ by:

$$
\begin{aligned}
& x \oplus y:=\max (x, y) \\
& x \otimes y:=x+y
\end{aligned}
$$

Definition 1: The set $\mathbb{R}_{\max }$ with the operations $\oplus$ and $\otimes$ is called the max-plus algebra, denoted by $\mathcal{R}_{\max }=$ $\left(\mathbb{R}_{\max }, \oplus, \otimes, \varepsilon, e\right)$.

Theorem 1: [2] The max-plus algebra $\mathcal{R}_{\max }$ has the algebraic structure of a commutative idempotent semiring.

The max-plus algebra can be interpreted as the traditional linear algebra with the operations ' + ' and ' $x$ ' replaced by the operators 'max' and ' + ', respectively, with the supplemental difference that the additive inverse does not exist, thus resulting in a semiring. Matrices can be defined by taking Cartesian products of $\mathbb{R}_{\max }$. Define the matrix sum $\oplus$, matrix product $\otimes$, and matrix power operations by:

$$
\begin{aligned}
{[A \oplus B]_{i j} } & =a_{i j} \oplus b_{i j}:=\max \left(a_{i j}, b_{i j}\right) \\
{[A \otimes C]_{i j} } & =\bigoplus_{k=1}^{m} a_{i k} \otimes c_{k j}:=\max _{k=1, \ldots, m}\left(a_{i k}+c_{k j}\right) \\
D^{\otimes k} & :=\underbrace{D \otimes D \otimes \ldots \otimes D}_{k \text {-times }},
\end{aligned}
$$

where $A, B \in \mathbb{R}_{\max }^{n \times m}, C \in \mathbb{R}_{\max }^{m \times p}, D \in \mathbb{R}_{\max }^{n \times n}$, and the $i, j$ element of $A$ is denoted by $a_{i j}=[A]_{i j}$. In this context, the max-plus zero $\mathcal{E}$, "one" $\mathbb{1}$, and identity $E$ matrices are defined by:

$$
\begin{aligned}
{[\mathcal{E}]_{i j} } & =\varepsilon \\
{[\mathbb{1}]_{i j} } & =e \\
{[E]_{i j} } & = \begin{cases}e & \text { if } i=j \\
\varepsilon & \text { otherwise. }\end{cases}
\end{aligned}
$$


We use the following notation to illustrate the dimensions of the previous matrices:

$$
\mathcal{E}_{n \times m} \in \mathbb{R}_{\max }^{n \times m} ; \quad \mathbb{1}_{n \times m} \in \mathbb{R}_{\max }^{n \times m} ; \quad E_{n} \in \mathbb{R}_{\max }^{n \times n}
$$

Finally, we define $D^{\otimes 0}:=E$ and $x^{\otimes 0}:=e$. Now let

$$
A^{*}:=\bigoplus_{k=0}^{\infty} A^{\otimes k}
$$

If $A^{*}$ exists then the vector $x=A^{*} \otimes b$ solves the system of max-plus linear equations

$$
x=A \otimes x \oplus b,
$$

with $A \in \mathbb{R}_{\max }^{n \times n}$ and $b \in \mathbb{R}_{\max }^{n}$ (see [2], Theorem 3.17). The matrix $D \in \mathbb{R}_{\max }^{n \times n}$ is called nilpotent if

$$
\exists k<\infty, \forall p>k: D^{\otimes p}=\mathcal{E}
$$

It is always the case that if $D$ is nilpotent then $k<n$.

Definition 2: The (square) matrix $A$ is called irreducible if no permutation matrix $B$ exists such that the matrix $\bar{A}$, defined by

$$
\bar{A}=B^{T} \otimes A \otimes B
$$

has an upper triangular block structure (an alternative definition states that a matrix $A$ is irreducible if its communication graph is strongly connected [3]).

Eigenvectors $\lambda$ and eigenvalues $v$ are defined in the same way as in the traditional algebra, where $v \neq \mathcal{E}$ :

$$
A \otimes v=\lambda \otimes v
$$

For max-plus linear systems the eigenvalue of the system matrix represents the total cycle time, whereas the eigenvector dictates the steady-state behavior.

Theorem 2: [2] If $A$ is irreducible, there exists one and only one eigenvalue (but possibly several eigenvectors).

Definition 3: For $A, B \in \mathbb{R}_{\max }^{n \times m}$ We say that matrix $A$ overcomes $B$, written as $A \geq B$ if $\forall(i, j) \in\{1, \ldots, n\} \times$ $\{1, \ldots, m\}$ we have:

$$
[A]_{i, j} \geq[B]_{i, j}
$$

In this situation we get that $A \oplus B=A$.

\section{MODELING LEGGED LOCOMOTION}

We model legged locomotion by abstracting the continuous-time motion of the legs into discrete-event cycles. Let $l_{i}(k)$ be the time instant leg $i$ lifts off the ground and $t_{i}(k)$ be the time instant it touches the ground, both for the $k$-th event index. Here, $k$ is considered to be a global event counter. For a traditional alternating swing/stance ${ }^{1}$ gait one can impose that the time instant when the leg touches the ground must equal the time instant it lifted off the ground for the last time plus the time it stays in flight (denoted by $\tau_{f}$ ):

$$
t_{i}(k)=l_{i}(k)+\tau_{f}
$$

Analogously, we get a similar relation for the lift-off time:

$$
l_{i}(k)=t_{i}(k-1)+\tau_{g},
$$

where $\tau_{g}$ is the stance time and $t_{i}$ uses the previous event index such that equations (2) and (3) can be used iteratively. Suppose now that one aims to synchronize leg $i$ with leg $j$ in such a way that leg $i$ can only lift off $\tau_{\Delta}$ seconds after leg $j$ has touched the ground ( $\tau_{\Delta}$ is the double stance time). One can then write the relation:

$$
\begin{aligned}
l_{i}(k) & =\max \left(t_{i}(k-1)+\tau_{g}, t_{j}(k-1)+\tau_{\Delta}\right) \\
& =\left[\begin{array}{ll}
\tau_{g} & \tau_{\Delta}
\end{array}\right] \otimes\left[\begin{array}{c}
t_{i}(k-1) \\
t_{j}(k-1)
\end{array}\right] .
\end{aligned}
$$

Equation (4) enforces simultaneously that both the leg $i$ stays at least $\tau_{g}$ seconds in stance and will only lift off at least $\tau_{\Delta}$ seconds after leg $j$ has touched down. When both conditions are satisfied, lift-off takes place. Following this reasoning, one can efficiently represent motion gaits in terms of synchronization of timed events.

For an $n$-legged robot, let the full discrete-event state vector be defined by:

$$
x(k)=[\underbrace{t_{1}(k) \cdots t_{n}(k)}_{t(k)} \underbrace{l_{1}(k) \cdots l_{n}(k)}_{l(k)}]^{T} .
$$

The $2 n$-dimensional system equations for the cycles represented by equations (2),(3) take the form:

$$
\begin{aligned}
{\left[\begin{array}{c}
t(k) \\
l(k)
\end{array}\right]=} & {\left[\begin{array}{c|c}
\mathcal{E} & \tau_{f} \otimes E \\
\hline \mathcal{E} & \mathcal{E}
\end{array}\right] \otimes\left[\begin{array}{l}
t(k) \\
l(k)
\end{array}\right] } \\
& \oplus\left[\begin{array}{c|c}
E & \mathcal{E} \\
\hline \tau_{g} \otimes E & E
\end{array}\right] \otimes\left[\begin{array}{c}
t(k-1) \\
l(k-1)
\end{array}\right]
\end{aligned}
$$

According to (5) all legs follow the same rhythm, i.e. all legs rotate with the same period of at least $\tau_{f}+\tau_{g}$ seconds. The introduction of the extra identity matrices $E$ in (5) results in the extra trivial constraints $t(k+1) \geq t(k)$ and $l(k+1) \geq$ $l(k)$. This enforces however, that the resulting system matrix will be irreducible (see last section of the proof of Theorem $3)$.

We assume that all leg synchronizations are achieved by enforcing a relation between the next lift-off time of a leg with the touchdown time of other legs (as in equation (4)). This assumption is expressed by the additional matrices $P$

\footnotetext{
${ }^{1}$ The biology and robotics communities use the terms leg "swing" and "stance" to denote when a leg is in flight or is touching the ground supporting the body, respectively. "Double stance" represents two legs touching the ground. In this paper we use the term double stance to denote when more than one leg is in stance.
} 
and $Q$ (that we define next) added to equation (5), resulting in the synchronized system:

$$
\begin{aligned}
{\left[\begin{array}{c}
t(k) \\
l(k)
\end{array}\right]=} & {\left[\begin{array}{c|c}
\mathcal{E} & \tau_{f} \otimes E \\
\hline P & \mathcal{E}
\end{array}\right] \otimes\left[\begin{array}{c}
t(k) \\
l(k)
\end{array}\right] } \\
& \oplus\left[\begin{array}{c|c}
E & \mathcal{E} \\
\hline \tau_{g} \otimes E \oplus Q & E
\end{array}\right] \otimes\left[\begin{array}{r}
t(k-1) \\
l(k-1)
\end{array}\right](6)
\end{aligned}
$$

which one can write using simplified notation as:

$$
x(k)=A_{0} \otimes x(k) \oplus A_{1} \otimes x(k-1) .
$$

Lemma 1: [10] A sufficient condition for $A_{0}^{*}$ to exist is that the matrix $P$ is nilpotent in the max-plus sense.

Equation (7) can be written explicitly by

$$
\begin{aligned}
x(k) & =A_{0}^{*} \otimes A_{1} \otimes x(k-1) \\
& =A \otimes x(k-1),
\end{aligned}
$$

where $A=A_{0}^{*} \otimes A_{1}$ is called the system matrix.

For a robot with $n$ legs let $\ell_{1}, \ldots, \ell_{m}$ be sets of integers such that

$$
\begin{aligned}
& \bigcup_{p=1}^{m} \ell_{p}=\{1, \ldots, n\}, \text { and } \\
& \forall i \neq j, \ell_{i} \cap \ell_{j}=\emptyset
\end{aligned}
$$

i.e., each set $\ell_{p}$ takes elements of $\{1, \ldots, m\}$ with no overlap between sets. Define $r_{p}=\# \ell_{p}$. We consider that each $\ell_{p}$ contains the indices of a set of legs that recirculate simultaneously (i.e. liftoff together). A gait $\mathcal{G}$ is defined as an ordering relation of groups of legs:

$$
\mathcal{G}=\ell_{1} \prec \ell_{2} \prec \cdots \prec \ell_{m}
$$

This ordering relation is interpreted in the following manner: the set of legs indexed by $\ell_{i+1}$ swings immediately after all the legs $\ell_{i}$ have reached stance arriving from their own swing. For example, a trotting gait on a quadruped robot where the legs are sorted by front-left, front-right, back-left, back-right, is represented by:

$$
\{1,4\} \prec\{2,3\}
$$

Given the previous notation, the matrices $P$ and $Q$ in equation (6) can be generated by: $\forall j \in\{1, \ldots, m-1\}, \forall p \in$ $\ell_{j+1}, \forall q \in \ell_{j}$

$$
[P]_{p, q}=\tau_{\Delta}
$$

and $\forall p \in \ell_{1}, \forall q \in \ell_{m}$

$$
[Q]_{p, q}=\tau_{\Delta},
$$

where all other entries of $P$ and $Q$ are $\varepsilon$. For example in the trotting gait defined above we get:

$$
P=\left[\begin{array}{cccc}
\varepsilon & \varepsilon & \varepsilon & \varepsilon \\
\tau_{\Delta} & \varepsilon & \varepsilon & \tau_{\Delta} \\
\tau_{\Delta} & \varepsilon & \varepsilon & \tau_{\Delta} \\
\varepsilon & \varepsilon & \varepsilon & \varepsilon
\end{array}\right] \text { and } Q=\left[\begin{array}{cccc}
\varepsilon & \tau_{\Delta} & \tau_{\Delta} & \varepsilon \\
\varepsilon & \varepsilon & \varepsilon & \varepsilon \\
\varepsilon & \varepsilon & \varepsilon & \varepsilon \\
\varepsilon & \tau_{\Delta} & \tau_{\Delta} & \varepsilon
\end{array}\right] \text {. }
$$

Define the function $b$ that transforms a gait into a vector of integers (here we assume that the ordering (9) is represented as a set of sets):

$$
\begin{aligned}
b: & \left\{\left[\ell_{1}\right]_{1}, \ldots,\left[\ell_{1}\right]_{i_{1}}\right\} \prec \cdots \prec\left\{\left[\ell_{m}\right]_{1}, \ldots,\left[\ell_{m}\right]_{i_{m}}\right\} \mapsto \\
& {\left[\left[\ell_{1}\right]_{1}, \ldots,\left[\ell_{1}\right]_{i_{1}} \ldots\left[\ell_{m}\right]_{1}, \ldots,\left[\ell_{m}\right]_{i_{m}}\right]^{T} }
\end{aligned}
$$

For example $b(\{1,4\} \prec\{2,3\})=\left[\begin{array}{llll}1 & 4 & 2 & 3\end{array}\right]^{T}$.

Definition 4: A gait $\overline{\mathcal{G}}$ is called a normal gait if the elements of the vector $b(\overline{\mathcal{G}})$ are sorted increasingly.

For a gait $\mathcal{G}$, define the similarity matrix $C$ such that:

$$
C=\left[\begin{array}{ll}
\bar{C} & \mathcal{E} \\
\mathcal{E} & \bar{C}
\end{array}\right]
$$

where $\forall i, j \in\{1, \ldots, n\}$ :

$$
[\bar{C}]_{i, j}= \begin{cases}e & \text { if }[b(\mathcal{G})]_{i}=j \\ \varepsilon & \text { otherwise }\end{cases}
$$

As an example, for the gait $\mathcal{G}=\{1,4\} \prec\{2,3\}$ we obtain:

$$
\bar{C}=\left[\begin{array}{llll}
e & \varepsilon & \varepsilon & \varepsilon \\
\varepsilon & \varepsilon & \varepsilon & e \\
\varepsilon & e & \varepsilon & \varepsilon \\
\varepsilon & \varepsilon & e & \varepsilon
\end{array}\right]
$$

The similarity matrix $C$ is such that

$$
C \otimes C^{T}=C^{T} \otimes C=E
$$

Moreover, $C$ transforms the system matrix $A$ of an arbitrary gait $\mathcal{G}$ into the system matrix $\bar{A}$ of a normal gait $\overline{\mathcal{G}}$ via the similarity:

$$
\bar{A}=C \otimes A \otimes C^{T}
$$

Such transformation is very useful since, by effectively switching lines and columns in $A$, one obtains a very structured matrix $\bar{A}$ where analysis is much simpler. Thus, the interpretation of the similarity matrix $C$ is that we can always rename the legs in a manner that simplifies the calculus. The matrices $P$ and $Q$ can also be transformed into their "normal" counterparts via:

$$
\begin{aligned}
& \bar{P}=\bar{C} \otimes P \otimes \bar{C}^{T} \\
& \bar{Q}=\bar{C} \otimes Q \otimes \bar{C}^{T}
\end{aligned}
$$

The structure of the matrices $\bar{P}$ and $\bar{Q}$ is illustrated by equations (22) and (23), respectively.

\section{EIGENSTRUCTURE OF THE SYSTEM MATRIX}

Let $\tau_{\delta}=\tau_{f} \otimes \tau_{\Delta}$ and consider the following assumptions (which are always satisfied in practice):

$$
\begin{array}{ll}
\text { A1 } & \tau_{g}, \tau_{f}>0 ; \quad \tau_{\Delta} \geq 0 \\
\text { A2 } & \tau_{g} \otimes \tau_{f} \leq \tau_{\delta}^{\otimes m}
\end{array}
$$

Theorem 3: If assumptions A1, A2 verify then the matrix $A$ defined by equations (8), (6) has a unique eigenvalue 
$\lambda=\tau_{\delta}^{\otimes m}$ and an eigenvector $v$ (up to scaling factor) defined by

$$
\forall j \in\{1, \ldots, m\}, \forall q \in \ell_{j}: \quad \begin{array}{ll} 
& {[v]_{q}=\tau_{f} \otimes \tau_{\delta}^{\otimes j-1}} \\
& {[v]_{q+n}=\tau_{\delta}^{\otimes j-1} .}
\end{array}
$$

Proof: Let $[\bar{v}]_{q}=[v]_{q+n}$ for all $j$ and $q \in \ell_{j}$. One can see that $v=\left[\left(\tau_{f} \otimes \bar{v}\right)^{T} \bar{v}^{T}\right]^{T}$. We now show that $\lambda$ and $v$ are an eigenvalue and eigenvector of $A$, respectively. Replacing the state variable $x(k-1)$ by $v$ and $x(k)$ by $\lambda \otimes v$ in equation (7), a necessary and sufficient condition for $\lambda$ and $v$ to be an eigenvalue and an eigenvector of $A$ is:

$$
\begin{aligned}
\lambda \otimes v & =\lambda \otimes A_{0} \otimes v \oplus A_{1} \otimes v \\
\Rightarrow \quad \lambda \otimes v & =\underbrace{A_{0}^{*} \otimes A_{1}}_{A} \otimes v
\end{aligned}
$$

Let us show that expression (12) indeed holds. We expand the matrices $A_{0}$, and $A_{1}$ to obtain:

$$
\begin{aligned}
& \lambda \otimes v=\lambda \otimes\left[\begin{array}{c}
\tau_{f} \otimes \bar{v} \\
\bar{v}
\end{array}\right]= \\
& \lambda \otimes\left[\begin{array}{c|c}
\mathcal{E} & \tau_{f} \otimes E \\
\hline P & \mathcal{E}
\end{array}\right] \otimes v \oplus\left[\begin{array}{c|c}
E & \mathcal{E} \\
\hline \tau_{g} \otimes E \oplus Q & E
\end{array}\right] \otimes v= \\
& {\left[\begin{array}{c|c}
E & \lambda \otimes \tau_{f} \otimes E \\
\hline \lambda \otimes P \oplus \tau_{g} \otimes E \oplus Q & E
\end{array}\right] \otimes\left[\begin{array}{c}
\tau_{f} \otimes \bar{v} \\
\bar{v}
\end{array}\right]}
\end{aligned}
$$

The previous expression results in the following two equations:

$$
\begin{aligned}
\lambda \otimes \tau_{f} \otimes \bar{v} & =\tau_{f} \otimes \bar{v} \oplus \lambda \otimes \tau_{f} \otimes \bar{v} \\
\lambda \otimes \bar{v} & =\tau_{f} \otimes\left(\lambda \otimes P \oplus \tau_{g} \otimes E \oplus Q\right) \otimes \bar{v} \oplus \bar{v}
\end{aligned}
$$

Since $\lambda>0$, equation (13) is always verified, thus one needs only to address equation (14), which can be simplified due to $\tau_{f} \otimes \tau_{g}>0$ :

$$
\lambda \otimes \bar{v}=\left(\tau_{f} \otimes \tau_{g}\right) \otimes \bar{v} \oplus \tau_{f} \otimes(\lambda \otimes P \oplus Q) \otimes \bar{v}
$$

If $\tau_{g} \otimes \tau_{f}>\lambda$ then the previous equation does not hold true. Therefore we consider only the case when $\tau_{g} \otimes \tau_{f} \leq \lambda$ (i.e. assumption A2) and focus on the right-hand term of equation (15) to obtain the simpler expression:

$$
\lambda \otimes \bar{v}=\tau_{f} \otimes(\lambda \otimes P \oplus Q) \otimes \bar{v}
$$

If (16) is verified, then (15) is also true. Let $\tau_{\Delta} \otimes P_{0}=P$ and $\tau_{\Delta} \otimes Q_{0}=Q$, i.e., all entries of matrices $P_{0}$ and $Q_{0}$ are either 0 or $\varepsilon$. Since $\lambda=\tau_{\delta}^{\otimes m}$ and $\tau_{\delta}=\tau_{f} \otimes \tau_{\Delta}$, we obtain:

$$
\tau_{\delta}^{\otimes m} \otimes \bar{v}=\tau_{\delta} \otimes\left(\tau_{\delta}^{\otimes m} \otimes P_{0} \oplus Q_{0}\right) \otimes \bar{v} .
$$

We now consider two cases:

i) First we analyze the row indices of equation (17) that are elements of the sets $\ell_{2}, \ldots, \ell_{m}$. I.e., $\forall j \in\{1, \ldots, m-1\}$ and $\forall p \in \ell_{j+1}$, for each row $p$ we obtain (notice that accordingly to (11) all the elements of $\left[Q_{0}\right]_{p,}$, are $\varepsilon$ since $p \notin \ell_{1}$, and that $[\bar{v}]_{p}=\tau_{\delta}^{\otimes j}$ for $\left.p \in \ell_{j+1}\right)$ :

$$
\begin{aligned}
{\left[\tau_{\delta}^{\otimes m} \otimes \bar{v}\right]_{p} } & =\tau_{\delta} \otimes\left[\tau_{\delta}^{\otimes m} \otimes P_{0} \oplus Q_{0}\right]_{p, \cdot} \otimes \bar{v} \Leftrightarrow \\
{\left[\tau_{\delta}^{\otimes m} \otimes \bar{v}\right]_{p} } & =\tau_{\delta} \otimes\left[\tau_{\delta}^{\otimes m} \otimes P_{0}\right]_{p, \cdot} \otimes \bar{v} \oplus[\underbrace{\left[Q_{0}\right]_{p, \cdot}}_{\varepsilon} \otimes \bar{v} \Leftrightarrow \\
\tau_{\delta}^{\otimes m} \otimes \tau_{\delta}^{\otimes j} & =\tau_{\delta} \otimes \bigoplus_{q \in \ell_{j}} \tau_{\delta}^{\otimes m} \otimes\left[P_{0}\right]_{p, q} \otimes[\bar{v}]_{q} \Leftrightarrow \\
\tau_{\delta}^{\otimes m+j} & =\tau_{\delta} \otimes \tau_{\delta}^{\otimes m} \otimes \tau_{\delta}^{\otimes j-1} \Leftrightarrow \\
\tau_{\delta}^{\otimes m+j} & =\tau_{\delta}^{\otimes m+j}
\end{aligned}
$$

Thus for rows $p$ equation (17) holds true.

ii) We now look at all the remaining rows $p$ such that $p \in \ell_{1}$ (noticing now that accordingly to (10) all the elements of $\left[P_{0}\right]_{p \text {, }}$ are $\varepsilon$ and that $[\bar{v}]_{p}=e$ since $\left.p \in \ell_{1}\right)$ :

$$
\begin{aligned}
{\left[\tau_{\delta}^{\otimes m} \otimes \bar{v}\right]_{p} } & =\tau_{\delta} \otimes\left[\tau_{\delta}^{\otimes m} \otimes P_{0} \oplus Q_{0}\right]_{p, \cdot} \otimes \bar{v} \Leftrightarrow \\
{\left[\tau_{\delta}^{\otimes m} \otimes \bar{v}\right]_{p} } & =\tau_{\delta} \otimes \underbrace{\left[\tau_{\delta}^{\otimes m} \otimes P_{0}\right]_{p, \cdot}}_{\varepsilon} \otimes \bar{v} \oplus\left[Q_{0}\right]_{p, \cdot} \otimes \bar{v} \Leftrightarrow \\
\tau_{\delta}^{\otimes m} & =\tau_{\delta} \otimes \bigoplus_{q \in \ell_{m}}\left[Q_{0}\right]_{p, q} \otimes[\bar{v}]_{q} \Leftrightarrow \\
\tau_{\delta}^{\otimes m} & =\tau_{\delta} \otimes \tau_{\delta}^{\otimes m-1} \Leftrightarrow \\
\tau_{\delta}^{\otimes m} & =\tau_{\delta}^{\otimes m}
\end{aligned}
$$

Combining i) and ii) we conclude that equation (17) holds true.

Irreducibility of the system matrix $\bar{A}$ (and its counterpart $A$ ) can be readily verified by inspection of (21) together with the expressions for $V$ (equation (25)) and $W$ (equation (24)): the $n-r_{m}$ to $n$ columns and rows of $\bar{A}$ are non- $\varepsilon$, and thus, any node of the graph can be reached by any other node via these columns and rows. Can can now take advantage of Theorem 2 to conclude that the eigenvalue is unique.

\section{Coupling time}

Theorem 4: [16] Let $A$ be an irreducible matrix. Then there exists $c \in \mathbb{N} \backslash\{0\}$ (the cyclicity of $A$ ), $\lambda \in \mathbb{R}$ (the unique eigenvalue of $A$ ), and $k_{0} \in \mathbb{N}$ (the coupling time of A) such that

$$
\forall k \geq k_{0} \quad A^{\otimes(k+c)}=\lambda^{\otimes c} \otimes A^{\otimes k}
$$

Theorem 4 describes an important property of max-pluslinear systems when the system matrix $A$ is irreducible: it guarantees the existence of a (uncontrolled) steady-state regime that can be achieved in a number of finite steps $k_{0}$, denoted the coupling time. Computing the coupling time is very important in our application since it gives the number of steps the robot needs to take to reach steady-state after a gait transition or a perturbation. The second main contribution of this paper comes in the form of the following lemma:

Lemma 2: The coupling time for the max-plus-linear system defined by equations (8), (6) is $k_{0}=2$ with cyclicity $c=1$. 
Proof: The coupling time is obtained by a laborious but straightforward set of computations. For an arbitrary gait $\mathcal{G}$ we compute the normal gait $\overline{\mathcal{G}}$ via the similarity transform $C$. From here on we use the normal system matrix $\bar{A}$ to compute the coupling time, culminating in the same result for the original matrix $A$. By observing the structures of $\bar{A}_{0}$ and $\bar{A}_{1}$ (derived from $\bar{P}$ and $\bar{Q}$ ) a closed form solution can be obtained for $\bar{A}_{0}^{*}$ :

$$
\bar{A}_{0}^{*}=\left[\begin{array}{cc}
W & \tau_{f} \otimes W \\
\bar{W} & W
\end{array}\right]
$$

where $W=\left(\tau_{f} \otimes \bar{P}\right)^{*}$ and $\bar{W}$ is such that $\tau_{f} \otimes \bar{W} \oplus E=W$. The structure of $W$ is illustrated in equation (24). An expression for $\bar{A}$ is then obtained:

$$
\begin{aligned}
\bar{A} & =\bar{A}_{0}^{*} \otimes \bar{A}_{1} \\
& =\left[\begin{array}{cc}
W & \tau_{f} \otimes W \\
\bar{W} & W
\end{array}\right] \otimes\left[\begin{array}{cc}
E & \mathcal{E} \\
\tau_{g} \otimes E \oplus \bar{Q} & E
\end{array}\right] \\
& =\left[\begin{array}{cc}
W \oplus \tau_{f} \otimes \tau_{g} \otimes W \oplus \tau_{f} \otimes W \otimes \bar{Q} & \tau_{f} \otimes W \\
\bar{W} \oplus \tau_{g} \otimes W \oplus W \otimes \bar{Q} & W
\end{array}\right]
\end{aligned}
$$

Let $V=W \otimes \bar{Q}$, illustrated by equation (25). One can show that:

$$
\begin{aligned}
W \otimes W & =W \\
W \otimes V & =V \\
V \otimes V & =\left(\lambda-\tau_{f}\right) \otimes V,
\end{aligned}
$$

where "-" in equation (20) is the traditional the minus sign. Since $\mu \otimes W \geq W$ for any $\mu>0$ the previous expression simplifies to:

$$
\bar{A}=\left[\begin{array}{cc}
\tau_{f} \otimes\left(\tau_{g} \otimes W \oplus V\right) & \tau_{f} \otimes W \\
\tau_{g} \otimes W \oplus V & W
\end{array}\right]
$$

Computing successive products of $\bar{A}$ and taking advantage of its structure and equations (18)-(20) one can write its $k$-th power $\bar{A}^{\otimes k}$, valid for all $k \geq 2$, illustrated by equation (26) (in this equation, the max-plus product sign $\otimes$ was dropped due to the limited printing space). By inspection of the expression of $\bar{A}^{\otimes k}$ in (26) one can observe that most terms are multiplying by a power of the eigenvalue $\lambda$. To factor out $\lambda$ of the matrix in (26) it is sufficient to show that $\tau_{f} \otimes V \otimes W \geq \tau_{f} \otimes \tau_{g} \otimes W$, i.e. all the terms of $\tau_{f} \otimes V \otimes W$ are larger then $\tau_{f} \otimes \tau_{g} \otimes W$. This can be confirmed in a straightforward fashion by inspecting equations (24) and (27). Taking advantage of this simplification one can obtain equations (28)-(30). Together with the similarity transformation we obtain the result valid for $k \geq 2$ :

$$
\begin{aligned}
A^{\otimes(k+1)} & =C \otimes \bar{A}^{\otimes k} \otimes C^{T} \\
& =C \otimes \lambda \otimes \bar{A}^{\otimes k} \otimes C^{T}=\lambda \otimes A^{\otimes k},
\end{aligned}
$$

thus concluding that the coupling time is $k_{0}=2$ with cyclicity $c=1$.

\section{CONCLUSIONS}

We have considered a class of max-plus linear systems for the synchronization of multiple two-state cycles, such as in the circumstance of legged locomotion in robotics, and have shown that its important structural properties can be obtained in closed-form. The eigenvalue of the system matrix represents the total cycle time, the eigenvector dictates the steady-state behavior, and the coupling time exposes the transient response. This result brings important insight into the modeling and control of legged locomotion systems where large numbers of legs result in a combinatorial gait space (due to all the possible combinations in which multiple legs can be synchronized). Further research is now taking place in relaxing the structure of the system matrix, towards addressing the synchronization of more general cyclic systems.

\section{REFERENCES}

[1] R. A. Cuninghame-Green, "Minimax algebra," Lecture Notes in Economics and Mathematical Systems, vol. 166, 1979.

[2] F. Baccelli, G. Cohen, G. Olsder, and J. Quadrat, Synchronization and Linearity: an Algebra for Discrete Event Systems. Wiley, 1992.

[3] B. Heidergott, G. Olsder, and J. van der Woude, Max Plus at Work: Modeling and Analysis of Synchronized Systems. Kluwer, 2006.

[4] J. G. Braker, "Max-algebra modelling and analysis of time-table dependent transportation networks," in Proc. of the First European Control Conf., 1991, pp. 1831-1836.

[5] B. Heidergott and R. Vries, "Towards a $(\max ,+)$ control theory for public transportation networks," Discrete Event Dynamic Systems, vol. 11, pp. 371-398, 2001.

[6] B. Heidergott, "A characterisation of (max,+)-linear queueing systems," Queueing Systems: Theory and Applications, vol. 35, pp. 237262, 2000.

[7] S. Gaubert and J. Mairesse, "Task resource models and (max,+) automata," in Idempotency, J. Gunawardena, Ed. Cambridge University Press, 1998.

[8] B. Bede and H. Nobuhara, "A novel max-plus algebra based wavelet transform and its applications in image processing," in IEEE Int. Conf. on Systems, Man and Cybernetics, 2009, pp. 2585 -2588.

[9] G. Lopes, R. Babuška, B. De Schutter, and T. van den Boom, "Switching max-plus models for legged locomotion," in Proc. of IEEE Int. Conf. on Robotics and Biomimetics, 2009, pp. 221-226.

[10] G. Lopes, T. van den Boom, B. De Schutter, and R. Babuška, "Modeling and control of legged locomotion via switching max-plus systems," in Proc. of the Int. Workshop on Discrete Event Systems, 2010, pp. 392-397.

[11] P. Holmes, R. Full, D. Koditschek, and J. Guckenheimer, "The dynamics of legged locomotion: models, analyses, and challenges," SIAM Review, vol. 48, no. 2, pp. 207-304, 2006.

[12] B. Giffler, "Mathematical solution of production planning and scheduling problems," IBM ASDD, Tech. Rep., Oct. 1960.

[13] R. Cuninghame-Green, "Describing industrial processes with interference and approximating their steady-state behaviour," Operational Research Quarterly, vol. 13, no. 1, pp. 95-100, 1962.

[14] G. Cohen, D. Dubois, J. Quadrat, and M. Viot, "A linear-systemtheoretic view of discrete-event processes and its use for performance evaluation in manufacturing," IEEE Transactions on Automatic Control, vol. 30, no. 3, pp. 210-220, 1985.

[15] S. Gaubert and M. Plus, "Methods and applications of $(\max ,+)$ linear algebra," in Proc. of Symp. on Theoretical Aspects of Computer Science, 1997, pp. 261-282.

[16] A. Bouillard and B. Gaujal, "Coupling time of a (max, plus) matrix," INRIA, Technical report 4068, 2000. 


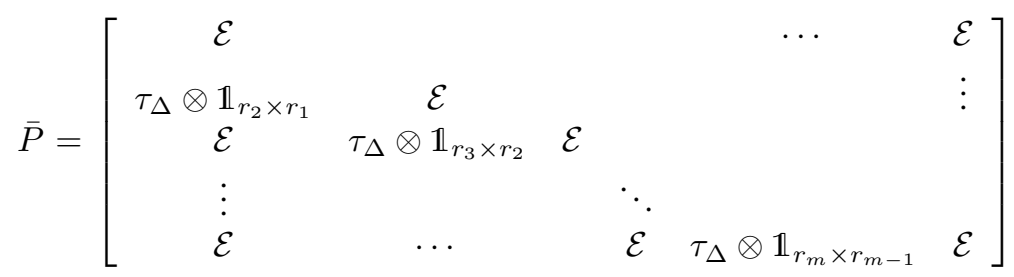

$$
\begin{aligned}
& \bar{Q}=\left[\begin{array}{cc}
\mathcal{E} & \tau_{\Delta} \otimes \mathbb{1}_{r_{1} \times r_{m}} \\
\mathcal{E} & \mathcal{E}
\end{array}\right]
\end{aligned}
$$

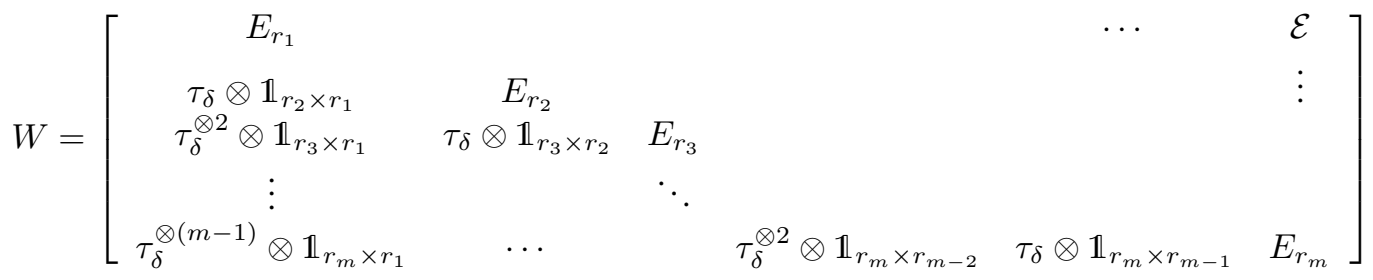

$$
\begin{aligned}
& V=\left[\begin{array}{c|c}
\tau_{\Delta} \otimes \mathbb{1}_{r_{1} \times r_{m}} \\
\mathcal{E}_{n \times\left(n-r_{m}\right)} \otimes \tau_{\delta} \otimes \mathbb{1}_{r_{2} \times r_{m}} \\
\vdots \\
\tau_{\Delta} \otimes \tau_{\delta}^{\otimes(m-1)} \otimes \mathbb{1}_{r_{m} \times r_{m}}
\end{array}\right] \\
& \bar{A}^{\otimes k}=\left[\begin{array}{cc}
\tau_{f}\left(\lambda^{\otimes(k-2)} \tau_{f} \tau_{g} V W \oplus \lambda^{\otimes(k-1)} V \oplus \tau_{f}^{\otimes(k-1)} \tau_{g}^{\otimes k} W\right) & \tau_{f}\left(\lambda^{\otimes(k-2)} \tau_{f} V W \oplus\left(\tau_{f} \tau_{g}\right)^{\otimes(k-1)} W\right) \\
\lambda^{\otimes(k-2)} \tau_{f} \tau_{g} V W \oplus \lambda^{\otimes(k-1)} V \oplus \tau_{f}^{\otimes(k-1)} \tau_{g}^{\otimes k} W & \lambda^{\otimes(k-2)} \tau_{f} V W \oplus\left(\tau_{f} \tau_{g}\right)^{\otimes(k-1)} W
\end{array}\right] \\
& \tau_{f} \otimes V \otimes W=\left[\begin{array}{ccc}
\tau_{\delta}^{\otimes m} \otimes \mathbb{1}_{n_{1} \times n_{1}} & \cdots & \tau_{\delta} \otimes \mathbb{1}_{n_{1} \times n_{m}} \\
\vdots & \ddots & \vdots \\
\tau_{\delta}^{\otimes(2 m-1)} \otimes \mathbb{1}_{n_{m} \times n_{1}} & \cdots & \tau_{\delta}^{\otimes m} \otimes \mathbb{1}_{n_{m} \times n_{m}}
\end{array}\right] \\
& \bar{A}^{\otimes(k+1)}=\left[\begin{array}{cc}
\tau_{f}\left(\lambda^{\otimes(k-1)} \tau_{f} \tau_{g} V W \oplus \lambda^{\otimes k} V\right) & \tau_{f}\left(\lambda^{\otimes(k-1)} \tau_{f} V W\right) \\
\lambda^{\otimes(k-1)} \tau_{f} \tau_{g} V W \oplus \lambda^{\otimes k} V & \lambda^{\otimes(k-1)} \tau_{f} V W
\end{array}\right] \\
& =\lambda \otimes\left[\begin{array}{cc}
\tau_{f}\left(\lambda^{\otimes(k-2)} \tau_{f} \tau_{g} V W \oplus \lambda^{\otimes(k-1)} V\right) & \tau_{f}\left(\lambda^{\otimes(k-2)} \tau_{f} V W\right) \\
\lambda^{\otimes(k-2)} \tau_{f} \tau_{g} V W \oplus \lambda^{\otimes(k-1)} V & \lambda^{\otimes(k-2)} \tau_{f} V W
\end{array}\right] \\
& =\lambda \otimes \bar{A}^{\otimes k}
\end{aligned}
$$

